\title{
Interfaces
}

\section{Manières de faire vernaculaires. Une introduction}

Jordi Ballesta et Eliane de Larminat

\section{(2) OpenEdition}

\section{Journals}

Édition électronique

URL : http://journals.openedition.org/interfaces/1453

DOI : $10.4000 /$ interfaces. 1453

ISSN : 2647-6754

\section{Éditeur :}

Université de Bourgogne, Université de Paris, College of the Holy Cross

\section{Édition imprimée}

Date de publication : 15 décembre 2020

Pagination : $9-28$

ISSN : 1164-6225

\section{Référence électronique}

Jordi Ballesta et Eliane de Larminat, « Manières de faire vernaculaires. Une introduction », Interfaces [En ligne], 44 | 2020, mis en ligne le 15 décembre 2020, consulté le 21 décembre 2020. URL : http:// journals.openedition.org/interfaces/1453; DOI : https://doi.org/10.4000/interfaces.1453

Les contenus de la revue Interfaces sont mis à disposition selon les termes de la Licence Creative Commons Attribution 4.0 International. 


\title{
MANIÈRES DE FAIRE VERNACULAIRES. UNE INTRODUCTION
}

\author{
Jordi Ballesta \\ Université Jean Monnet, CIEREC
}

Eliane de Larminat

Université de Paris, LARCA

Le mot vernaculaire n'est présent dans aucun des dictionnaires de l'Académie française, depuis sa première édition de 1694. Il l'est depuis 1994 dans le TLFI (Trésor de la Langue Française informatisé) où il est rattaché aux domaines de la didactique, de la linguistique et des sciences naturelles, présenté comme un synonyme d' «autochtone, domestique, indigène» et, enfin, défini comme ce qui est «propre à un pays, à des habitants», comme «la langue communément parlée dans les limites d'une communauté» - «par opposition à la langue véhiculaire» - et comme un «nom vulgaire d'animal ou de végétal, par opposition aux noms qui suivent les règles de la nomenclature scientifique $»^{1}$. Ainsi, le vernaculaire ne répond pas aux conventions officielles, tant il lie domesticité et expression populaire. Absent des dictionnaires de l'Académie, le mot vernaculaire l'est aussi du Littré, quand bien même ce terme possède une étymologie ancienne. Le TLFI indique qu'il est un dérivé du latin vernaculus «(propr. 'relatif aux esclaves nés dans la maison') 'du pays, indigène, national [vernacula vocabula]' " et constate l'emploi de vernacule («langue maternelle») dans Pantagruel de Rabelais. Pour autant, il semble improbable que son utilisation actuelle, croissante dans les champs de l'architecture, du paysage, du design et de la photographie, procède de la réactivation d'un sens endogène. Elle dérive plus certainement de la traduction du mot anglais vernacular.

Consulter les dictionnaires contemporains de langue anglaise laisse d'ailleurs apparaître des définitions à la fois plus précises et plus amples : «the language or dialect spoken by the ordinary people in a particular country or region [;] occurring in the everyday language of a place and regarded as native or natural to it [;] the term is used contrastively to compare the mainly or only oral expression of a people, a rural or urban community, or a lower social class [...] with languages and styles that are classical, literary, liturgical, or more socially and linguistically cultivated and prestigious [;] relating 
to the plain standard style or variety of a language as opposed to more ornate, pedantic, classical, or complex styles and varieties $[\ldots] »^{2}$. Aux significations qui ont cours en français, sont ajoutées celles qui informent une appartenance de classe, inférieure, une inscription dans le commun - de l' « ordinaire» et du «quotidien» - et une simplicité des formes. À l'emploi spécifique aux sciences naturelles se substituent plusieurs références à l'architecture, domaine qui, aux États-Unis et en Angleterre, s'avère un des plus fructueux dans la théorisation du vernaculaire.

Dans ce dossier, la notion de vernaculaire sera principalement étudiée à partir de démarches croisées, visant à interroger ses teneurs sémantiques et à questionner les manières de faire qu'elle permet d'identifier. Plus que l'architecture, ce seront des modes d'habitation et des pratiques d'origine domestique qui seront investis dans cette introduction et les articles suivants. Ce sont aussi les façons d'en rendre compte, et parmi elles de les documenter, qui seront explorées, avec une attention plus grande à la photographie. Nous supposons en effet que photographie et vernaculaire sont intimement liés, en raison de leur ouverture aux pratiques populaires, aux savoir-faire non conventionnels et au monde domestique ${ }^{3}$. Enfin, ce dossier, «Les manières de faire vernaculaires / Vernacular Ways», entend contribuer à un effort théorique renouvelé qui s'accompagne régulièrement d'une généalogie de la notion ${ }^{4}$. Dans le monde académique, le vernaculaire reste en effet une terre exotique, même si en tant que notion il relève du vocabulaire savant et si une partie, ou du moins un fragment de la recherche - principalement anglophone - en a fait son objet.

Dans cette introduction, nous commencerons par sonder sa définition progressive dans les études architecturales, les sciences sociales et l'histoire de la photographie.

2. Citations issues des définitions données par The Oxford Pocket Dictionary of Current English et Concise Oxford Companion to the English Language via la page web: https://www.encyclopedia.com/literature-and-arts/languagelinguistics-and-literary-terms/language-and-linguistics/vernacular (page consultée le 29 novembre 2020).

3. Outre les pratiques photographiques amateures, nous pensons, parmi une multitude d'exemples potentiels, aux photographies de William Fox Talbot, The Open Door et The Haystack, à la documentation de logis ouvriers par Jacob Riis, à une partie majeure de l'œuvre d'Eugène Atget et de Walker Evans, à An American Exodus de Dorothea Lange et Paul Taylor, à certains travaux de la mouvance «New Topographics» et à la photographie paysagère contemporaine, dès lors qu'elle s'intéresse aux espaces qui échappent à l'urbanisme et à l'aménagement du territoire.

4. Nous pensons notamment à l'ouvrage Learning from Vernacular de Pierre Frey (2010), à la thèse de doctorat d'Edith Hallauer, Du vernaculaire à la déprise d'œuvre. Urbanisme, architecture, design (2017), aux travaux à paraître de Jérôme Meizoz. La généalogie que nous dressons dans cette introduction est évidemment parcellaire, tant elle demanderait de plus amples développements, en incluant notamment les travaux de Lewis Mumford et ceux de Henry-Russell Hitchcock, commissaire en 1932 de l'exposition «The Urban Vernacular of the Forties, Fifties and Fifties: American Cities before the Civil War» (Mileaf 1993) structurée autour de photographies de Berenice Abbott. 
En 1981, Dell Upton, jeune historien de l'architecture et des cultures matérielles américaines, publie «Ordinary Buildings: A Bibliographical Essay on American Vernacular Architecture» (Upton). Il y répertorie plus de cent-cinquante livres, articles et revues parus depuis 1895. Thématique centenaire, le vernaculaire en architecture est toutefois l'objet d'investigations théoriques longtemps restées à l'état liminaire. Upton en donne une idée en avançant une définition succincte qui, écrit-il, dominait quelques années avant qu'il ne publie son essai. Il l'explicite, non pas en décrivant des manières de bâtir, mais en indiquant le premier élément d'une typologie architecturale qu'il amplifiera dès la phrase suivante. Jusqu' au milieu des années 1970, «un bâtiment traditionnel, rural, domestique et agricole ${ }^{5}$ était considéré comme l'archétype de l'architecture vernaculaire (57). Quand Upton écrit «Ordinary Buildings », la notion d'architecture vernaculaire sert selon lui à qualifier des fermes construites avant la révolution industrielle, des granges, des stands de hamburger, des maisons préfabriquées et montées sur place (tract houses). Elle embrasse aussi des paysages, qu'ils soient historiques comme ceux de Williamsburg et Lowell, qu'ils émanent d'une forme métropolitaine, celle de Los Angeles notamment, ou qu'ils s'étendent à l'échelle des aires suburbaines. Upton cite les lotissements de Levittown et les strips - avenues où s'accumulent les enseignes et où les consommateurs sont d'abord des automobilistes qui observent les commerces depuis leur véhicule en mouvement, avant de se garer sur un parking et descendre s'acheter des services et des biens.

En ce début des années 1980 pointent deux versants sémantiques. L'un fait côtoyer vernaculaire, ruralité pré-industrielle et autosubsistance (agricole); l'autre lie vernaculaire, fabrication industrielle et société de consommation. L'un invite à constater l'existence d'un patrimoine marginalisé par des productions architecturales et paysagères fondées sur la systématisation des procédés de construction et la généralisation de l'économie marchande. L'autre part au contraire de l'hypothèse que le vernaculaire occupe une place centrale dans les cultures contemporaine et populaire, que celles-ci soient strictement matérielles ou déterminent plus largement nos façons d'habiter.

Pour Upton, les «vernacular architecture studies» sont à la convergence de plusieurs disciplines. Il y reconnaît les travaux d'universitaires (scholars) mais aussi d'auteurs qui ont fait de la recherche sans en faire leur profession, parmi lesquels des historiens, des anthropologues, des archéologues, des folkloristes, des géographes, des américanistes, des acteurs du patrimoine, et bien sûr des architectes. Ces chercheurs, statutaires ou non, sont, selon lui, animés par une pensée égalitariste qui provient d'une considération commune pour «les cultures populaires» et repose sur un goût partagé pour «l'architecture non académique», « la vie quotidienne et les valeurs culturelles d'une vaste majorité d'Américains qui n'ont pas créé ou ne créent pas de grands monuments, ou ne laissent pas de longues analyses écrites 
sur leurs pensées et leurs actions» (57-58). Avec ces termes émerge une première définition, davantage ontologique que typologique, qui n'a plus comme modèle la ruralité traditionnelle et rejoint nombre des acceptions qui font aujourd'hui autorité, jusqu'à avoir été intégrées dans les dictionnaires anglophones : le vernaculaire embrasse des manières de faire qui ne sont pas élitistes, savantes, institutionnelles, qui génèrent peu de documents et participent de l'ordinaire - d'un ordinaire aussi bien matériel que symbolique (ce qui ne relève pas du grand et du monumental), aussi bien spatial que temporel (qui appartient à la vie quotidienne). En avançant vers cette définition, Upton met également de côté les réticences idéologiques qu'inspirent les cultures populaires au sein des études culturelles et patrimoniales américaines. Il en fera cas à plusieurs reprises, en citant notamment un de leurs principaux animateurs, Henry Glassie.

En 1968, Glassie publie Pattern in the Material Folk Culture of the Eastern United States et emploie le mot vernaculaire (vernacular) aux ultimes pages de son ouvrage, dans une note en bas de page. S'il trouve la notion fascinante, il refuse d'y voir un synonyme de folklorique (folk) - notion qu'il privilégie - et l'associe au mot populaire (Glassie 1971, 240). En cela, il se détourne de la proximité sémantique du vernaculaire et du folklorique que John A. Kouwenhoven avait instaurée en 1948, dans Made in America: The Arts in Modern Civilization - livre qui s'avère essentiel pour comprendre la portée, les orientations et l'actualité sémantiques du mot vernaculaire (Kouwenhoven 1948). A partir du chapitre «What is Vernacular? », Kouwenhoven avance l'existence d'un «art folklorique» (15) - traduction ici littérale de «folk art» - mais qui relève plutôt d'un art populaire, actuel et en l'occurrence proprement américain - autant de qualités incluses dans son acception du vernaculaire. Nationalisant cette notion, il la conçoit aussi comme un antonyme d' «européen». Il l'utilise comme le vecteur d'une opposition entre les valeurs esthétiques d'une société dont les raffinements littéraires et artistiques assoient son caractère élitiste et les valeurs pratiques d'un régime démocratique, reposant sur des savoir-faire pragmatiques, un esprit industrieux et porté vers le fonctionnalisme. Or, pour Glassie, si le vernaculaire épouse les normes d'une société américaine désormais pourvoyeuse de biens industriels et de culture populaire, il diverge alors du folklorique. «Normative», « de masse», définie par «l'élite» et pleinement acquise au consumérisme, la culture populaire se situe, pour lui, aux antipodes des cultures folkloriques. Celles-ci participent de «ce qui est inférieur à la norme [substandard]» (Glassie 1971, 28); elles sont produites par des communautés homogènes, autonomes et isolées qui se maintiennent à distance d'une «société dominée par une culture populaire approuvée politiquement et économiquement» $(4)^{6}$.

Notons que Glassie gommera ses préventions contre la notion de vernaculaire deux décennies plus tard. En 2000, il publiera Vernacular Architecture, ouvrage dans lequel, comme son titre l'indique, il s'approprie pleinement cette notion (Glassie 2000). 
Reconnaître ou exclure l'existence des cultures vernaculaires au sein des sociétés contemporaines, consuméristes et industrielles implique, de fait, de se positionner face à un schisme qui a probablement émergé durant la période de l'après-guerre, pris forme à partir des années 1960 et s'est cristallisé dans les années 1980, sans toutefois avoir donné lieu à de nombreuses confrontations directes, tant le vernaculaire était l'objet d'investigations théoriques isolées.

Bien qu'il ne se soit pas placé explicitement dans le sillage de Kouwenhoven, John Brinckerhoff Jackson pourrait avoir hérité de ses propositions théoriques ${ }^{7}$. En lançant en 1951 le magazine Landscape, il accorde une place croissante aux paysages routiers, qu'il voit comme l'émanation d'une culture proprement américaine, à la fois automobile, commerciale, graphique et consumériste. Si la notion de vernaculaire ne semble guère y être utilisée, Landscape ouvre la voie à des études de terrain, écrites et visuelles, et des propos théoriques sur ce qui fait l'ordinaire géographique mais échappe aux conceptions savantes et notamment esthétiques des architectes et urbanistes ${ }^{8}$. Son essai «Other-directed Houses» (Jackson 1957) annonce la publication quinze ans plus tard de Learning from Las Vegas, ouvrage qui consacre le strip comme une figure emblématique du vernaculaire américain (Venturi, Scott Brown et Izenour).

Pour autant, en 1957, la même année que cet essai, Sybil Moholy-Nagy avance de son côté une interprétation tout autre du vernaculaire. Elle publie Native Genius in Anonymous Architecture, dans lequel elle fait cohabiter vernaculaire, indigénat et primitivité. Loin de l'extraversion graphique et commerciale des strips, elle étudie et valorise des manières de bâtir fondées sur «l'usage exclusif de matériaux et de compétences de construction d'origine locales», sur «la planification comme résultat de conditions locales et d'exigences fonctionnelles impossibles à reproduire» et sur «l'absence d'ornements ne faisant pas partie de la structure» (Moholy-Nagy 44-5). D'un ton critique, Hilde Heynen écrira à propos de cet ouvrage qu'il est une manifestation limpide de «la fascination de nombreux architectes modernes et critiques pour les bâtiments exotiques, anonymes, autochtones ou vernaculaires qui résultent d'une supposée interaction non médiatisée et directe entre l'homme et son environnement» (Heyden 469).

Cette fascination et exotisation se retrouve chez Bernard Rudofksy qui, en 1964, présente au MoMA 1'exposition «Architecture Without Architects: An Introduction to Non-Pedigreed Archi-

Jackson, au vu de ses archives, et notamment des ouvrages qu'il avait conservés avant son décès, n'entretenait pas de liens scientifiques importants avec Kouwenhoven. Les témoignages collectés auprès de ses proches collègues tendent à le confirmer. Au stade actuel de nos recherches, seule une recension de The Beer Can by the Highway (Kouwenhoven 1961), publiée dans le magazine Landscape, laisse à penser que Jackson connaissait les travaux de Kouwenhoven.

8. Voir à ce sujet Notteboom (2018). 
tecture». Dans le catalogue qu'il publie parallèlement, Rudofsky indique s'être tourné vers «une architecture informelle [non-formal], inclassée [non-classified]» et s'être ouvert à un "monde non familier» (Rudofsky n.p.). L' «architecture sans architectes» qu'il examine est aussi «vernaculaire, anonyme, spontanée, indigène, rurale» ou encore «exotique»- mot qu'il emploie «dans son sens original, étranger [alien]». Cette architecture, il la trouve quasi exclusivement dans des contextes pré-industriels, médiévaux ou localisés dans des «pays prétendument non développés».

Cinq après, Paul Oliver, spécialiste du blues devenu également historien de l'architecture, édite et introduit l'ouvrage collectif Shelter and Society, dans lequel il remet en cause, mot par mot, le caractère spontané, anonyme, indigène et rural du vernaculaire, jusqu'à affirmer que l'interprétation rudofskienne est moraliste et puritaine (Oliver 21). Dans la continuité, Oliver définit le vernaculaire comme une qualité architecturale et plus largement un phénomène culturel qui n'est pas cantonné aux contrées exotiques, mais qui est aussi endogène aux sociétés industrielles et associé aux modes de vie consuméristes. À l'instar des publications articulant vernaculaire, autochtonie, tradition et folklore, Shelter and Society porte sur les maisons en bois laftehus en Norvège, les fermes bernoises en Suisse, les «maisons orientales» d'Irak, les villages Hopi en Arizona, et ceux de la Volta noire au Ghana, tout en ayant pour objet les manières de construire dans les bidonvilles péruviens, les quartiers populaires qui émergent dans les périphéries athéniennes et les dômes géodésiques de la communauté hippie de Drop City. Il qualifie d'ailleurs de «vernaculaire moderne véritable» (32) ces trois derniers exemples fondés sur l'auto-construction graduelle, quand les ressources le permettent, sur une part notable d'illégalité, au Pérou et à Athènes, et sur l'utilisation de matériaux industriels neufs ou récupérés, parmi lesquels la brique, le béton armé, et des pare-brise pour les habitants de Drop City. Trois décennies plus tard, Lindsay Asquith et Marcel Vellinga, héritiers d'Oliver à Oxford Brookes University, considéreront que les habitations vernaculaires «constituent environ 90\% du stock mondial de logements » (Asquith et Vellinga 1) - une telle statistique invitant à penser que le vernaculaire n'a pas été marginalisé à mesure que l'industrialisation, les pratiques de consommation et l'urbanisation se généralisaient. Cette assertion tend à confirmer que vernaculaire et ordinaire sont liés par une part majeure de synonymie.

À partir des années 1980, deux auteurs vont s'emparer de la notion de vernaculaire. Ils lui donneront une épaisseur sémantique et une portée sociologique qu'elle n'avait pas atteint depuis les deux livres de Kouwenhoven, Made in America (1948) puis The Beer Can by the Highway (1961). Il s'agit d'Ivan Illich et à nouveau de John Brinckerhoff Jackson, deux incarnations d'une recherche, l'une davantage philosophique, l'autre davantage géographique, qui, plus que d'établir une définition du vernaculaire, vont en donner une acception rhizomatique, s'amplifiant au fil de leurs argumentations.

D'un côté, Illich publie en 1981 Shadow Work et sa traduction Le Travail fantôme, puis en 1983 Le Genre vernaculaire, traduction de Gender paru un an plus tôt outre-Atlantique (Illich 1981 et 
1983). Dans le premier ouvrage, le vernaculaire est défini comme un domaine indépendant de l'économie industrielle et marchande, et de ce qu'elle implique comme corvées non monétarisées mais nécessaires à la production, l'acquisition, la consommation de biens et services standardisés. Exemple typique de ce travail fantôme: prendre la voiture, remplir son réservoir d'essence, se garer au parking pour se rendre au supermarché, mettre dans un chariot des plats préparés dont il faudra scanner soi-même le code-barre pour les acheter. À ce travail qui a perdu en domesticité, en étant réduit à des tâches ménagères et en reposant sur la consommation de produits extérieurs, Illich oppose le vernaculaire, qu'il conçoit comme le domaine où l'homo habilis assure sa subsistance, en mobilisant ses compétences généralistes et en menant une pluralité d'activités quotidiennes qui ne sont pas planifiées, hiérarchisées et contrôlées par un organe institutionnel (Illich 1981). Pour Illich, le vernaculaire disparaît en même temps que l'homo industrialis intègre à son mode de vie la standardisation et la division des tâches, notamment à travers ces deux déclinaisons: « vir laborans, le travailleur, et femina domestica, la femme au foyer» (23).

De l'autre, Jackson s'approprie la notion de vernaculaire à partir de la conférence «Houses and Trailers » qu'il prononce à Harvard en 1979 (Jackson 2016a). Cette notion deviendra le fil conducteur de la plupart de ses recherches ultérieures. Après Kouwenhoven, il l'américanisera en écrivant de nombreux essais et interventions publiques, parmi lesquels «Vernacular Space», « Vernacular Gardens», « The Vernacular City», « Urban Circumstances », « The Popular Yard», « Working at Home», « The Mobile Home and the Range» et son livre le plus influent, Discovering the Vernacular Landscape, paru en 1984 et traduit en français dix-neuf ans après (Jackson 2003). Mais plus qu'une américanisation supplémentaire du vernaculaire, Jackson en propose une analyse croisant géographie, approches historiques, études culturelles et architecturales. En 1986, il s'identifie à un dénommé «Kevin», figure archétypique du manœuvre sans patrimoine ni contrat de travail stable, auto-construisant sa maison en acquérant et récupérant des matériaux industriels, ne pouvant se tenir à des plans établis mais saisissant toutes les opportunités passagères pour aboutir, in fine, à des conditions raisonnables d'habitation (Jackson 2016b). Le vernaculaire selon Jackson et selon Illich voisine avec la notion de domestique. Il qualifie un domaine qui échappe aux règles édictées par la sphère politique, aux normes créées et validées par ses institutions, de même qu'aux savoir-faire des professionnels. En revanche, chez l'auteur de «Houses and Trailers », le vernaculaire ne s'efface pas à mesure que l'industrialisation progresse et que les activités de subsistance laissent place à l'achat de marchandises. Il se greffe sur la société de consommation, réinterprète ses principes, $\mathrm{y}$ insère des manières de faire de type dialectal ${ }^{9}$, en somme il ouvre des espaces d'appropriation à même d'accroître temporairement ou à la marge le capital limité des classes populaires. En cela, il se

9. Si Bruno Zevi n'a pas fait du vernaculaire une notion centrale de sa théorie architecturale, il a écrit sur des manières de faire similaires, notamment dans Dialectes architecturaux (Zevi). 
rapproche des arts de faire, activités de braconnage et ruses de consommateurs décrits par Michel de Certeau (1990); il loge une part de communs entre les domaines public et privé - communs qui servent de ressources complémentaires aux besoins d'habitation et qui, occasionnant un débordement de la sphère domestique, réduisent conséquemment la sphère de pouvoir des institutions politiques.

Au vernaculaire selon Jackson et selon Illich peuvent être articulées des pensées plus récentes, qui s'appuient ou non sur la notion et comportent des dimensions plutôt sociologiques, géographiques, écologiques, etc., mais toujours politiques. Pensons à La Ville vue d'en bas où le collectif Rosa Bonheur analyse la production des centralités populaires et ce qu'elles embrassent comme formations sur le tas, auto-constructions, stockages et activités économiques à la fois non déclarées et développées au sein de l'espace public. Pensons à la multitude de réflexions pratiques et théoriques sur les communs ou à la manière dont la notion de tiers-paysage de Gilles Clément peut être conjuguée avec celle de vernaculaire. Envisager que ce «fragment indécidé du jardin planétaire» ne soit pas délaissé par l'homme permet ainsi de concevoir l'existence d'un paysage tiers et vernaculaire, «n'exprimant ni le pouvoir ni la soumission au pouvoir » (Clément 1), qu'il soit politique ou institutionnel. Pensons également aux thèses libertaires et décentralisatrices de James C. Scott qui voit «dans les trois derniers siècles le triomphe des paysages de contrôle standardisés et officiels et leur mainmise sur l'ordre vernaculaire» et qui oppose, d'un côté, la disposition des lieux dans une franchise McDonald's «calculée jusque dans ses moindres détails, de manière à maximiser le contrôle du matériel et des méthodes de travail depuis le centre» (Scott 2019, 78), et de l'autre, la fertilité des imbrications spatiales et interrelations botaniques développées «en dépit, ou plutôt en raison de ce que la vision occidentale du jardinage perçoit comme un désordre visuel» $(98)^{10}$. La «vision occidentale» de Scott, nous pourrions ici l'interpréter en tant qu'une modernité planificatrice, dans ce qu'elle engendre d'établissements, d'ordonnancements et de programmations, et en ce qu'elle s'oppose aux agencements complexes et domestiques que génère le vernaculaire.

Scott a insisté sur l'opacité du vernaculaire face à la volonté de connaissance et à la rationalisation scientifiques et étatiques, opacité qui est aussi souvent une résistance à l'écriture (Scott 2002). Justement, alors que l'étude des paysages et constructions vernaculaires s'est largement appuyée sur la photographie (Ballesta et Fallet; Stierli; Esperdy), cette notion de résistance à l'écriture invite à considérer dans quelle mesure des pratiques visuelles d'enregistrement ont participé (et peuvent participer) de sa connaissance. Articuler une histoire visuelle au vernaculaire selon Jackson et selon Illich implique de considérer les images à la fois comme le lieu d'une pratique vernaculaire, et comme vecteur d'accès au vernaculaire, de sa reconnaissance voire de sa patrimonialisation sans dénaturation.

10. James C. Scott s'appuie ici sur les travaux menés au Guatemala par Edgar Anderson, botaniste qui fut, par ailleurs, un des collaborateurs les plus réguliers de la revue Landscape. 
Cela impose une double focale, comme celle adoptée par Jackson dans le seul texte publié que ce chercheur, qui pratiquait lui-même la photographie régulièrement en amateur à des fins de connaissance géographique, a consacré à la photographie. Dans son avant-propos au livre de photographies Joe Deal: Southern California Photographs, Jackson présentait, d'une part, une vision populaire de la photographie comme médium permettant, depuis l'origine, des pratiques non spécialistes et relevant d'utilisateurs (users) plutôt que de producteurs (producers), et, d'autre part, la capacité d'auteurs non anonymes et formés dans un cadre académique (Joe Deal était passé d'une formation universitaire à l'enseignement universitaire de la photographie) à donner à voir des réalités sociales et paysagères non standardisées (Jackson 1981). En l'occurrence, il s'agissait, dans de nouveaux lotissements, d'une appropriation «ouvrière» du paysage, par l'habitat et par la superposition de l'auto-construction au préfabriqué. Fidèle à son intérêt pour les «utilisateurs», Jackson achevait son essai sur l'espoir que la photographie d'un Joe Deal enrichirait le répertoire thématique de la photographie ordinaire. Un tel texte appelle en tout cas à penser les rapports entre photographie vernaculaire et photographie du vernaculaire, qui correspondent aux deux manières dont la notion de vernaculaire est prise en charge dans le champ des études visuelles.

$* * *$

Depuis une vingtaine d'années, les références muséales et universitaires à des images dites «vernaculaires» (familiales, commerciales, amateures, etc.) relèvent avant tout de réflexions d'ordre pratique et théorique sur l'extension des corpus exposés, conservés et étudiés, face aux opportunités mais aussi aux défis relatifs à l'intégration de nouvelles masses d'images. Si les pratiques d'exposition ont pu impliquer des mécanismes de sélection de «certaines images remarquables» en consonance avec les préoccupations d'une photographie artistique ou une vision préétablie et esthétisante de la photographie amateur $^{11}$, l'ouverture programmatique des corpus dans les disciplines qui prennent la photographie pour objet - en histoire de l'art, études visuelles, anthropologie ou études matérielles - fait écho à l'ouverture du champ considéré par les études sur l'architecture dès lors qu'une définition renouvelée du vernaculaire s'y est établie. La thèse selon laquelle la quasi-totalité du bâti est vernaculaire trouve un équivalent dans l'idée que la quasi-totalité des images photographiques (cartes postales, albums familiaux ou snapshots) n'est pas produite par des artistes ou spécialistes formés dans un cadre académique. Alors que l'idée d'un vernaculaire contemporain a conduit les architectes à porter attention à des modes de construction d'un faible niveau technique et employant des matériaux industriels, le vernaculaire en photographie invite à embrasser la totalité des images issues d'une pratique populaire

11. Sur ce point, et sur le fait que le renouvellement des objets demande aussi un renouvellement des méthodes, voir l'article de Carolin Görgen dans ce dossier. 
fondée sur l'appropriation d'un procédé mécanique qui, à compter du développement de la photographie instantanée dans les années 1880, ne requiert plus de compétences professionnelles.

En 2000, 1'historien de la photographie Geoffrey Batchen lance un appel à étudier la photographie vernaculaire dans un essai dont le titre, «Vernacular Photographies», associe durablement les deux termes (Batchen). Il souligne la forme particulièrement aiguë qu'a prise la dichotomie art contre vernaculaire dans les études photographiques, compte tenu de puissantes logiques de légitimation muséale pour ce médium; il suggère que les images vernaculaires sont traitées comme un parergon - «cette chose qui détermine ce que la bonne photographie [proper photography] n'est pas» (59) et appelle à une remise en cause de cette opposition en histoire et critique de la photographie, que ce soit en pensant une vernacularité de l'art ou en accordant aux images vernaculaires le sérieux et l'attention qui est accordé à l'art dans les humanités (76). Il y a pourtant débat sur la centralité de l'art pour la notion de photographie vernaculaire; Clément Chéroux, qui définira premièrement le vernaculaire comme «utilitaire, domestique et hétérotopique», écrira en 2013 que «la photographie n'est vernaculaire que quand elle tend un miroir à l'art» (Chéroux 2013, 10 et 14). Sa définition du vernaculaire évoluera néanmoins, puisque Chéroux proposera en 2017 que le vernaculaire est «utile, domestique et populaire» (Chéroux 2017, 10), substituant à l'idée d'une extériorité à l'art (le vernaculaire comme «hétérotopique») une caractéristique sociale et endogène (le vernaculaire comme «populaire»).

En fait, plutôt que d'un tournant vernaculaire, on peut parler d'une histoire des pratiques du médium qui est restée marginale par rapport aux histoires centrées sur une définition esthétique du médium - ces histoires que François Brunet a qualifiées d' «internalistes » ${ }^{12}$ (Brunet 2017a). Aux États-Unis, un auteur comme Robert Taft - chimiste et producteur d'une histoire «externaliste» et non institutionnelle de la photographie abordée sous un double angle technique et social, à qui Brunet a consacré des recherches pionnières dans Photographie. Histoire et contre-histoire, pense dès les années 1930 la masse des usages photographiques comme relevant du régime technologique-démocratique que Kouwenhoven qualifiera de vernaculaire en 1948. Ce dernier a d'ailleurs prononcé en 1972 et 1973 une conférence sur l'esthétique de la photographie instantanée, intitulée «Living in a Snapshot World», dont le texte sera publié dans le recueil Half a Truth Is Better Than None (Kouwenhoven 1982, 147-81). Il n'utilise pas dans cet essai le mot «vernaculaire», mais le texte s'ouvre sur la description illustrée d'un album photo (149-57), objet qui sera au cœur de travaux qui donneront à l'étude de la

12. François Brunet oppose une perspective «internaliste» centrée sur la photographie conçue comme un des beaux-arts, qui est celle d'un Beaumont Newhall dans les années 1930, à une approche «externaliste» qui relie les photographies à l'histoire événementielle et sociale, qu'il associe à Walter Benjamin mais aussi à Robert Taft (Brunet 2017a). 
photographie vernaculaire le statut d'un champ académique interdisciplinaire au tournant des années 2000 (voir Batchen; voir aussi Edwards ; Langford) ${ }^{13}$.

Une autre manière d'introduire du jeu dans l'opposition entre art et vernaculaire consiste à considérer principalement la photographie comme un médium d'enregistrement, une fonction élémentaire que l'on trouve dans les pratiques populaires et appliquées, mais aussi dans beaucoup de pratiques photographiques relevant du champ artistique. L'intérêt que des auteurs, artistes ou chercheurs, portent à des cultures matérielles, des architectures et des paysages vernaculaires permet de faire entrer ces derniers au musée, dans des galeries, des livres, des archives, etc. (Chéroux 2017; Ballesta et Fallet). Il est d'ailleurs significatif que deux des premières associations, sinon les deux premières associations, des mots photographie et vernaculaire concernent les sujets documentés par deux artistes photographes: Berenice Abbott et Walker Evans.

En 1934, Abbott expose cinquante photographies, qu'elle a produites en collaboration avec l'historien de l'architecture Henry-Russell Hitchcock, dans le cadre de l'exposition «The Urban Vernacular of the Thirties, Forties and Fifties ». Hitchcock, qui a publié deux ans plus tôt The International Style: Architecture since 1922 avec Philip Johnson (Hitchcock et Johnson) et qui s'affirme comme une figure majeure dans la théorisation du Mouvement moderne, introduit l'objet de l'exposition en décrivant un bâti urbain ordinaire construit avant la guerre de sécession, le plus souvent anonyme et aux datations incertaines (Mileaf 62).

Evans a aussi photographié des architectures domestiques de la période victorienne en 1933 pour une exposition d'architecture; mais surtout, comme l'a établi Anne Bertrand, le terme «vernaculaire» apparait dans ses écrits peu avant sa rencontre avec Kouwenhoven au début des années 1960. Il s'en sert alors pour décrire les formes d'architecture qu'il photographie (il évoque aussi une décoration intérieure «ni professionnelle, ni consciente»), et ensuite seulement, en 1964, pour qualifier des images anonymes qui l'inspirent (en l'occurrence les cartes postales) (Bertrand 40-41). Pour sa part, Kouwenhoven écrit en 1961 à propos des sujets d'Evans qu'ils relèvent d'un «design naturel» que lui-même tient à appeler «vernaculaire», autrement dit des agencements spatiaux «populaire[s]» et «improvisé[s]» (41). En 1971, Evans déclarera à l'éditeur Leslie Katz: «Je m’intéresse à ce qu'on appelle le vernaculaire» (Evans 35). À partir de son répertoire de sujets photographiques, des arrangements domestiques aux enseignes commerciales, on peut établir avec Evans que le vernaculaire moderne est une culture matérielle qui est aussi, consubstantiellement, une culture visuelle.

13. Les articles d'Elizabeth Edwards et de Geoffrey Batchen et l'ouvrage de Martha Langford sont publiés respectivement en 1999, 2000 et 2001. Les albums photos sont l'objet de nombreuses études depuis deux décennies; voir l'article de Carolin Görgen dans ce dossier. 
Ces termes, «architecture» et «décoration intérieure», «populaire» et «improvisé», nous convient à revenir à l'immersion documentaire de Walker Evans et de l'écrivain James Agee chez des métayers du Hale County (Alabama) en 1936, bien avant cette conceptualisation naissante, dans les années 1960 et 1970, d'une photographie tournée vers le vernaculaire. Le reportage d'Evans et Agee en pleine crise des années 1930 devient un livre en 1941, avec Let Us Now Praise Famous Men: Three Tenant Families (Agee et Evans) - titre paradoxal qui amplifie par avance le thème de la célébration du commun dans l'intérêt porté aux phénomènes vernaculaires dans la deuxième moitié du $\mathrm{XX}^{\mathrm{e}}$ siècle (Wright). Cette œuvre nous intéresse ici parce qu'elle dégage de façon à la fois précoce et radicale une veine vernaculaire liée à la précarité plus qu'à l'ordinaire. En photographiant des familles aux conditions de vie misérables dans leurs habitations de bois brut, Evans enregistre la forme éminemment visible que prend le métayage comme système d'exploitation agricole et sociale. Dans l'édition de $1960^{14}$, le portfolio d'images qui précède le texte d'Agee inclut la photographie d'une tombe d'enfant réduite à un maigre monticule, l'inverse d'un monument, figurant un passage sur terre qui ne laisse pas de trace durable - la «terre» étant ici très concrètement celle de la culture du coton et de la propriété agricole dont les métayers sont de simples occupants temporaires (n.p.). Par ailleurs, alors que l'espace domestique des trois familles de métayers est marqué par la fragilité des constructions et par une esthétique de l'ascétisme, qui a le manque pour principe directeur, Evans collecte photographiquement quelques objets décoratifs sur des cheminées environnées d'images punaisées au mur, inscription momentanée d'une mémoire familiale et d'aspirations sociales. L'association de photographies de famille et d'images commerciales (calendriers publicitaires illustrés ou ornements promotionnels comme des silhouettes d'animaux en fer blanc), dans ces zones de l'habitation qui sont faites pour être vues, témoigne aussi de la pénétration de ces logis de peu par une culture populaire «de masse». Enfin, dans un célèbre passage du livre, Agee place la photographie au-dessus du texte, comme forme de documentation la plus proche des matières périssables et presque informes dont est fait le quotidien: «Si je le pouvais, à ce point je n'écrirais rien du tout. Il y aurait des photographies; pour le reste, des morceaux d'étoffe, des déchets de coton, des grumelons de terre, des paroles rapportées, des bouts de bois, des pièces de fer, des fioles d'odeurs, des assiettées de nourriture et d'excrément» (30).

Le Hale County, transformé en monument documentaire par Agee et Evans, est resté un territoire vernaculaire durablement arpenté depuis 1936 - que ce soit par des photographes faisant œuvre documentaire, comme William Christenberry à partir des années 1960 (Southall), ou par des architectes travaillant en marge de l'institution académique, comme le Rural Studio qui depuis 1993 produit des logements pour et avec une population pauvre à partir de la récupération des rebuts de la

14. Une deuxième édition du livre est publiée en 1960. En plus d'une préface d'Evans, elle inclut une version révisée et augmentée du portfolio photographique. 
société industrielle (Oppenheimer; Goodman). Au-delà d'un territoire géographique spécifique, il existe un «domaine du vernaculaire» illustré par le Hale County, qui correspond à un paysage ré-organisé, ré-agencé de façon plus ou moins momentanée autour d'existences individuelles et familiales, et qui se prête à des entreprises d'enregistrement photographique attentives aux configurations spatiales et aux traces laissées par l'activité quotidienne des groupes sociaux les plus précaires.

À partir de son souvenir d'un quartier populaire d'une petite ville du Kentucky, bell hooks publie en 1994 un texte intitulé «In Our Glory: Photography and Black Life» (hooks 1994). Elle y lance un appel à une histoire des pratiques photographiques africaines-américaines, qu'elle ancre dans la tradition des «murs d'images» qui rassemblaient des portraits photographiques (snapshots ou portraits de studios commerciaux) dans les maisons noires du Sud ségrégué des États-Unis. Elle évoque en particulier la prolifération de portraits exposés chez sa propre grand-mère comme exemple de pratiques photographiques (voire curatoriales) contre-hégémoniques, à l'envers de l'institution raciste. Hooks n'emploie pas dans ce texte précis le terme «vernaculaire»; son essai semble pourtant anticiper, depuis une position radical black feminist et dans le cadre d'une histoire «faite maison», l'appel lancé six ans plus tard par Geoffrey Batchen en faveur d'une histoire qui se fonde sur l'affect, les pratiques mémorielles, la matérialité des images au sein de l'environnement domestique conçu comme espace extra-institutionnel de la photographie (Batchen). En 1995, hooks inclut «In Our Glory» dans le recueil d'essais Art on My Mind, qui porte sur une «politique de la race» également entendue comme une «politique de la culture», et en particulier d'une culture vernaculaire noire «working-class» contre la «haute culture» (hooks 1995, 89) - lecture de classe qui fait écho à la définition d'un vernaculaire ouvrier («blue collar») par Jackson (Ballesta 2016). Un autre essai de hooks intitulé «Black Vernacular: Architecture as Cultural Practice» évoque dans ce même recueil, et à nouveau à partir de souvenirs des maisons de ses grands-parents dans le Sud ségrégué, un rapport vernaculaire à l'habitation (hooks 1995). Hooks décrit des «shacks », logis en bois surpeuplés et fragiles, dont les habitants ne sont souvent pas propriétaires, mais qui sont décorés, organisés, modifiés, étendus par l'occupation d'espaces liminaux, l'aménagement du backyard ou du porch, etc. Ces pratiques spatiales provisoires mais créatives relèvent selon l'auteure d'une esthétique noire vernaculaire; parlant en tant qu'intellectuelle africaine-américaine, elle appelle à «la documentation de notre rapport historique et contemporain à l'espace et à l'esthétique» (151). Si dans ces deux essais c'est le texte qui se prête à l'évocation de souvenirs et de logements disparus (ici ceux des grands-parents), la sphère domestique et le vernaculaire sont conjointement associés aux manifestations visibles (et documentables) d'une agentivité - avec des configurations matérielles même peu marquées, qui sont à la fois le lieu et le produit d'une pratique et qui se voient: «the planting of flowers, the positioning of a porch or a rope-hung swing» (149).

Ces détours par des modes d'habitation prolétaires dans le Sud des États-Unis, en introduction d'un dossier qui accordera une part importante au domestique et au voisinage (traduction littérale de 
l'anglais «neighborhood»), permettent de dégager non seulement une coïncidence matérielle mais aussi une proximité d'intérêts documentaires (à la fois artistiques et intellectuels) entre, d'une part, des pratiques visuelles ordinaires, familiales, situées (dans des cultures plus ou moins locales et dans des lieux concrets), et, d'autre part, des manières d'habiter quotidiennement - ces dernières donnant lieu à des matérialisations, à un marquage géographique plus ou moins conscient et durable, et plus ou moins visuel et visible.

Bien sûr, si le mot «vernaculaire» est étymologiquement lié à «l'esclave né à la maison», les environnements qu'il décrit ne se réduisent ni au lumpenproletariat contemporain («l'esclave»), ni au domestique comme sphère distincte et bien circonscrite («à la maison»). Le «fait maison» n'est pas forcément voué à rester dans la maison, ni le local à rester en place, ainsi que le suggère d'ailleurs le lien, aux États-Unis, entre l'histoire des cultures matérielles vernaculaires et les transferts et adaptations liés à l'immigration (Upton 65-7). Néanmoins, si l'on considère que le logis est l'un des espaces où se joue la singularité au sein même d'une culture massifiée, en suivant par exemple l'hypothèse des «arts de faire» de Michel de Certeau (1980) (c'est-à-dire l'idée que les pratiques quotidiennes individuelles, et en particulier les manières d'employer les produits industriels consommés, permettent une dé-standardisation), alors l'attention pour cet environnement participe d'un intérêt plus large pour les interventions individuelles (ou émanant de groupes spécifiques) qui prennent place dans l'espace commun et modifient l'espace public. Ces modifications fragmentaires, qui ne sont pas concertées politiquement ni régies par des conventions ayant valeur de règlement, peuvent inclure tout aussi bien la prolifération des enseignes commerciales selon des initiatives privées discontinues et hors de toute planification urbanistique, qui fut un des sujets étudiés par Jackson (aussi en les photographiant) comme le furent également les modes d'habitation en mobile homes. C'est en effet en s'attachant, au sens large, "aux habitats et aux habitants, des formes bâties aux symboles qui les environnent et les caractérisent» (Brunet 2017b), entre régularités et occurrences singulières, que des entreprises de documentation visuelle (qu'elles soient le fait d'artistes ou de chercheurs) participent à rendre compte des pratiques vernaculaires. Une histoire visuelle, et notamment photographique, se grefferait ainsi au vernaculaire selon Jackson et selon Illich. Elle commencerait là où les administrations tendent à ne plus produire de documentation écrite sur les réalités sociales et géographiques; là où le monde académique choisit d'enregistrer par l'image parce qu'il ne parvient pas à décrire précisément par le texte; quand les archives et collections muséales intègrent des productions conçues comme amateurs ou domestiques, ou conservent visuellement des constructions et objets fabriqués qui ne donnent pas lieu à la constitution d'un patrimoine matériel. 
Le dossier «Les manières de faire vernaculaires / Vernacular Ways », qui comprend cinq articles et un entretien en plus de la présente introduction et de la photographie de couverture par Camille Fallet (une image qui documente une construction on ne peut plus vernaculaire), propose certains éléments d'une telle histoire visuelle. Il considère à la fois des images vernaculaires et des images du vernaculaire, des œuvres d'artistes et des pratiques de chercheurs, des habitats et des habitants.

Dans son article «Everyday Photography? Politicizing a 'vernacular' photo album of the San Francisco Earthquake and Fire of 1906 », Carolin Görgen prend pour objet un album photographique anonyme, constitué juste après la catastrophe de 1906 à partir d'images prises au milieu des ruines, et déposé peu de temps après à la California Historical Society. Elle montre comment, de la prise de vue au séquençage et au légendage des images dans l'album, un photographe-habitant inscrit une mémoire civique et familiale, mémoire à inflexion distinctement anti-élitaire, dans un paysage urbain dévasté puis dans le patrimoine de la ville. En l'occurrence, la destruction du paysage politique se prête à une prise de contrôle momentanée et mineure de l'espace physique et symbolique, qui trouve une forme de pérennisation dans une archive locale - à la condition que soit aujourd'hui pris au sérieux l'ancrage socio-politique de ce type de collection photographique.

En analysant la production photographique de l'architecte montréalais Melvin Charney dans son article «De l'Américanité à la Montréalité», Elène Levasseur fait ressortir bien plus qu'une pratique amateure, développée en parallèle d'une activité professionnelle prépondérante. À partir de l'étude de ses archives, elle montre comment Charney a fait de la photographie une écriture documentaire lui permettant de penser la contemporanéité de l'architecture québécoise et des formes urbaines de sa ville. Elle reconstitue les liens que Charney a tissés avec les œuvres photographiques de l'architecte Erich Mendelsohn et de l'artiste conceptuel Edward Ruscha, tout en nous informant de ses échanges avec Walker Evans dans le cadre du séminaire que celui-ci dirigeait à l'université de Yale. Par ailleurs, son article donne à voir près de dix images de Charney, uniquement visibles dans le fichier pdf $^{15}$, représentatives d'une articulation étroite entre style documentaire et documentation du vernaculaire.

Dans son article «La photographie: médium d'une exploration du logement, espace de syntaxes domestiques et provisoires », Danièle Méaux examine parallèlement trois ouvrages photographiques intitulés Intérieurs, qui sont unis par des formes comparables d'enquête et une même problématique: comment des habitants disposent leur mobilier, décorent leur logis, inscrivent leur vie quotidienne et organisent des «syntaxes privées». À partir de ces trois livres de François Hers, Jean-Marc Tingaud et Hortense Soichet, qui couvrent trois décennies de photographies, elle met en tension habitation et

15. À la demande des ayants droit. 
agencements précaires, aliénation consumériste et arts de faire, en envisageant la part de vernaculaire que comportent les mondes domestiques ordinaires.

Quittant l'image fixe pour l'image animée, toutes deux enregistrées, ce dossier s'oriente vers des pratiques cinématographiques et vidéographiques qui donnent une large place au vernaculaire comme caractéristique paysagère et comme mode de représentation. Dans son article «Agnès Varda à Los Angeles et l'hypothèse d'une forme cinématographique du vernaculaire», Lucas Lei interroge d'abord la manière dont la théorie cinématographique s'est appropriée la notion de vernaculaire et comment la pensée paysagère caractérise les cadres de vie urbains des Américains ordinaires. Il analyse ensuite deux films, le documentaire Murs murs et la fiction empreinte de réalisme Documenteur, qu'Agnès Varda a tournés à Los Angeles, dans des quartiers situés en contre-bas des studios hollywoodiens. Il y décèle une réflexivité entre le vernaculaire de la forme cinématographique et celui du paysage filmé - domestique dans Documenteur, jalonné de fresques murales et urbaines dans Murs murs.

Dans son article «Du vernaculaire comme genre cinématographique», Gala Hernández López avance une généalogie de la notion de vernaculaire à partir des études visuelles, de la philosophie et au sein de la littérature vidéographique, afin d'interroger les vidéos créées par des «produsers», à la fois producteur et consommateurs-utilisateurs non-professionnels, qui sont diffusées sur les plateformes d'hébergement dédiées, de YouTube à Periscope. Elle questionne la position de ces vidéos dans l'économie visuelle de masse actuelle, leur circulation au regard du régime d'autorialité qui leur est conféré, puis leur appropriation par des artistes faisant avec des matériaux qu'ils insèrent dans leurs œuvres. Ainsi, Gala Hernández López avance l'hypothèse d'un double mouvement de vernacularisation des formes vidéographiques puis de leur artialisation, aboutissant à un stade paradoxal de canonisation par les institutions muséales.

En conclusion de ce dossier, nous avons souhaité donner la parole au sociologue et photographe Jerome Krase qui, depuis plusieurs décennies, utilise l'observation flottante afin d'analyser les évolutions paysagères causées par l'immigration, la mondialisation et la gentrification au sein de quartiers populaires, notamment à Brooklyn et dans des villes polonaises et italiennes. Attentif aux déploiements graphiques et matériels qui ont cours à l'interface des espaces public et privé, il a créé une vaste archive visuelle témoignant d'un art de la notation des moins normés et des plus directs, qui, dans le même temps, documente une multitude d'activités immobilières, d'agencements mobiliers, de publicités commerciales et de pratiques jardinières qu'il qualifie de vernaculaire. Enfin, revenant sur l'usage académique de cette notion et les manières de faire qu'elle sous-tend, Jerome Krase invite à l'investir dans le cadre des sciences sociales. 


\section{Ouvrages CITÉS}

AGEE, James et Walker EVANS. Louons maintenant les grands hommes. 1960. Trad. de l'anglais par Jean Queval. Paris: Plon, 1972.

ASQUITH Lindsay et Marcel Vellinga, dir. Vernacular Architecture in the $21^{\text {st }}$ Century: Theory, Education and Practice. Abindgon-on-Thames: Taylor \& Francis, 2005.

BALLESTA, Jordi. «Le vernaculaire selon John: Quatre étapes dans la définition d'une notion centrale dans l'œuvre de J. B. Jackson». Carnets du paysage 30 («John Brinckerhoff Jackson» 2016) : 31-45.

BALLESTA, Jordi et Camille FALLET. Notes sur l'asphalte: Une Amérique mobile et précaire, 1950-1990. Vanves: Hazan, 2017.

BATCHEN, Geoffrey. «Vernacular Photographies». Each Wild Idea: Writing Photography History. Cambridge, Massachusetts: MIT Press, 2000. 56-80.

BERTRAND, Anne. «'I'm a writer too.' Les textes de Walker Evans». Walker Evans. Dir. Clément Chéroux. Catalogue d'exposition. Paris: Centre Pompidou, 2017. 39-44.

BRUNET, François. La Photographie. Histoire et contre-histoire. Paris : Presses universitaires de France, 2017a.

BRUNET, François. «Walker Evans, photographe vernaculaire? ». Métropolitiques (19 octobre 2017b). URL: https://www.metropolitiques.eu/Walker-Evans-photographe-vernaculaire.html (page consultée le 28 novembre 2020$)$.

CERTEAU, Michel de. L'invention du quotidien. 1. Arts de faire. 1980. Paris: Gallimard (Folio), 1990.

CHÉROUX, Clément. Vernaculaires. Essais d'histoire de la photographie. Paris: Le Point du Jour, 2013.

CHÉROUX, Clément, dir. Walker Evans. Catalogue d'exposition. Paris : Centre Pompidou, 2017.

CLEMENT, Gilles. Manifeste du Tiers-Paysage. Paris : Sujet/objet, 2014.

COLLECTIF ROSA BONHEUR. La ville vue d'en bas. Travail et production de l'espace populaire. Paris: Éditions Amsterdam, 2019.

EDWARDS, Elizabeth. «Photographs as Objects of Memory». Material Memories: Design and Evocation. Dir. Marius Kwint, Christopher Breward et Jeremy Aynsley. Oxford et New York: Berg, 1999. 221-236.

ESPERDY, Gabrielle. Autopia: An Intellectual History of the American Roadside at Midcentury. Charlottesville: University of Virginia Press, 2019.

EVANS, Walker. Le Secret de la photographie. Entretien avec Leslie Katz. 1971. Trad. de l'anglais par Bernard Hoepffner. Édition établie par Anne Bertrand. Paris : Éditions du centre Pompidou, 2017. 
FREY, Pierre. Learning from Vernacular. Arles: Actes Sud, 2010.

GLASSIE, Henry. Pattern in the Material Folk Culture of the Eastern United States. 1968. Philadelphie: University of Pennsylvania Press (Publications in folkore and folklife), 1971.

GLASSIE, Henry. Vernacular Architecture. Philadelphie: Material Culture; Bloomington: Indiana University Press, 2000.

GOODMAN, Anna G. «The Paradox of Representation and Practice in the Auburn University Rural Studio». Traditional Dwellings and Settlements Review 25.2 (printemps 2014): 39-52.

HALLAUER Edith, Du vernaculaire à la déprise d'œuvre. Urbanisme, architecture, design. Thèse de doctorat en urbanisme, Université Paris Est, 2017.

HEYDEN, Hilde. «Anonymous Architecture as Counter Image: Sibyl Moholy-Nagy’s Perspective on American Vernacular». The Journal of Architecture 13.4 (2008): 469-491.

HITCHCOCK, Henry Russell et Philip JOHNSON. The International Style: Architecture since 1922. New York: W. W. Norton \& company, 1932.

HOOKS, bell. «In Our Glory: Photography and Black Life». Picturing Us: African American Identity in Photography. Dir. Deborah Willis. New York: The New Press, 1994. 43-53.

HOOKS, bell. «Black Vernacular: Architecture as Cultural Practice». Art on My Mind: Visual Politics. New York: The New Press, 1995. 145-151.

ILLICH, Ivan. Le Travail fantôme. 1981. Trad. de l'anglais par Maud Sissung. Paris: Le Seuil, 1981.

ILLICH, Ivan. Le Genre vernaculaire. 1982. Trad. de l'anglais par Maud Sissung. Paris : Le Seuil, 1983.

JACKSON, John. B. «Other-directed Houses». 1957. Landscape: Selected Writings by J. B. Jackson. Amherst, Massachusetts: The University of Massachusetts Press, 1970.

JACKSON, John. B. «Maisons et caravanes». 1979. Trad. de l'anglais par Jordi Ballesta. Les Carnets du paysage 30 («John Brinckerhoff Jackson» 2016a): 126-151.

JACKSON, John. B. «Joe Deal and the Vernacular». Joe Deal: Southern California Photographs. Albuquerque: University of New Mexico Press, 1992. 3-7.

JACKSON, John. B. À la découverte du paysage vernaculaire. 1984. Trad. de l'anglais par Xavier Carrère. Arles, Actes Sud et Versailles, ENSP, 2003.

JACKSON, John. B. «Conférence $1 »$. 1986. Trad. de l'anglais par Henry Colomer. Les Carnets du paysage 30 («John Brinckerhoff Jackson» 2016b) : 152-165. 
KOUWENHOVEN, John A. Made in America: The Arts in Modern Civilization. Garden City et New York: The Country Life Press, 1948.

KOUWENHOVEN, John A. The Beer Can by the Highway: Essays on What's "American” about America. Baltimore: The John Hopkins University Press, 1961.

KOUWENHOVEN, John Attlee. «Living in a Snapshot World». Half a Truth Is Better Than None: Some Unsystematic Conjectures about Art, Disorder, and American Experience. Chicago: Chicago University Press, 1982. 147-181.

LANGFORD, Martha. Suspended Conversations: The Afterlife of Memory in Photographic Albums. Montréal: McGill-Queen's University Press, 2001.

MILEAF, Janine A. Constructing Modernism, Berenice Abbott and Henry-Russel Hitchcock: A Re-creation of the 1934 Exhibition, The Urban Vernacular of the Thirties, Forties and Fifties: American Cities Before Civil War. Middletown, Connecticut: The Center, 1993.

MOHOLY-NAGY, Sybil. Native Genius in Anonymous Architecture. New York: Horizon Press, 1957.

OLIVER, Paul, dir. Shelter and Society. New York et Washington, D.C.: Frederick A. Praeger publishers, 1969.

OPPENHEIMER, Dean Andrea et Timothy HURSLEY. Rural Studio: Samuel Mockbee and an Architecture of Decency. Princeton: Princeton Architectural Press, 2002.

RUDOFSKY, Bernard. Architecture Without Architects: An Introduction to Non-Pedigreed Architecture. New York: The Museum of Modern Art, 1964.

SCOTT, James C., John TEHRANIAN et Jeremy MATHIAS. «The Production of Legal Identities Proper to States: The Case of the Permanent Family Surname». Comparative Studies in Society and History 44.1 (janvier 2002): 4-44.

SCOTT, James C. Petit éloge de l'anarchisme. 2012. Trad. de l'anglais par Patrick Cadorette et Miriam HeapLalonde. Montréal: Lux éditions, 2019.

SOUTHALL, Thomas. Of Time and Place: Walker Evans and William Christenberry. Albuquerque: University of New Mexico Press, 1990.

STIERLI, Martino. Las Vegas in the Rearview Mirror: The City in Theory, Photography, and Film. Trad. de l'allemand par Elizabeth Tucker. Los Angeles: Getty Research Institute, 2013.

UPTON, Dell. «Ordinary Buildings: A Bibliographical Essay on American Vernacular Architecture». American Studies International 19.2 (hiver 1981): 57-75.

VENTURI, Robert, Denise SCOTT BROWN et Steven IZENOUR. Learning from Las Vegas. Cambridge: MIT Press, Cambridge, 1972. 
WRIGHT, Gwendolyn. «On Modern Vernaculars and J. B. Jackson». Geographical Review 88.4 (octobre 1998): 474-482.

ZEVI, Bruno. Dialectes architecturaux. Fermanville: Editions du Linteau, 2016. 